\title{
Factores que determinan la motivación por aprender en estudiantes universitarios
}

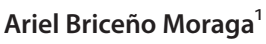

\section{INFORMACIÓN DEL ARTÍCULO}

Recibido: 30.04 .2020

Aprobado: 30.04 .2020

\section{Palabras claves:}

Motivación,

Factores Personales,

Pedagógicos,

Sociodemográficos

Factores Institucionales.

\section{RESUMEN}

El presente estudio investigativo tiene como propósito describir los principales factores que determinan la motivación por aprender en los estudiantes de los cuartos y quintos años de las carreras del Departamento de Ciencias Económicas y Administrativas de la Facultad Regional Multidisciplinaria de Chontales, de la UNAN - MANAGUA (UNAN - FAREM - Chontales), realizado entre el período del II Semestre del año 2017y I Semestre del 2018. El estudio se enmarcó en el enfoque mixto, el tipo y diseño de la investigación fue de carácter correlacional ya que examina la relación que existe entre los factores que determinan la motivación por aprender en los estudiantes en estudio, apoyado en una investigación documental y de campo. La población está compuesta por 266 estudiantes. La muestra total del estudio está conformada por 78 estudiantes. Se utilizó la encuesta como instrumento. La validez se obtuvo a través de la técnica del juicio de expertos, y la confiabilidad se determinó aplicando la prueba de dos mitades. El análisis de los datos se presenta en los cuadros y gráficos donde la información recabada se distinguió por frecuencia, las cuales fueron traducidos en porcentajes para representar las respuestas emitidas por los estudiantes.

\section{Factors that determine the motivation to learn in university students}

\begin{abstract}
The purpose of this research study is to describe the main factors that determine the students' learning motivation when they are in fourth and fifth level of their careers belonging to the Economic and Administrative Sciences Department, at the Regional Multidisciplinary Faculty of Chontales, UNAN - MANAGUA (UNAN - FAREM - Chontales). It was carried out between the II Semester of 2017 and I Semester of 2018. The study was framed in a mixed approach, the type and design of the research was correlational since it examines the relationship between the factors that determine the students' motivation for learning, supported by documentary and field research.
\end{abstract}

\footnotetext{
${ }^{1}$ Doctor en Gestión y Calidad de la Educación. Profesor de la Universidad Nacional Autónoma de Nicaragua, Managua - Facultad Regional Multidisciplenario - Chontales. E-mail: ariel.briceno7o@gmail.com 이: https://orcid.org/oooo-0001-9106-1391
} 
The population is composed by 266 students. The total sample of the study is made up of 78 students. The survey was used as an instrument. The validity was obtained through the technique of experts' judgment, and the reliability was determined by applying the two halves test. The analysis of data is presented in the tables and graphs where the collected information was distinguished by frequency. In addition, it was also translated into percentages to represent the responses issued by the students.

\section{Introducción}

La motivación representa un término amplio y complejo que incluye varias teorías y debe entenderse como un conjunto de procesos implicados en la activación, dirección y persistencia de la conducta orientada a lograr un objetivo o satisfacer una necesidad. En el ámbito educativo, la motivación se refiere a la disposición, que, de manera general, pueda tener el estudiante para aprender. Así mismo, Martínez-Salanova (s.f.) plantea que la motivación no debe confundirse con lo que el profesor hace para estimular y orientar el interés del estudiante hacia las tareas académicas.

Cabe destacar que un estudiante motivado logrará rendimientos académicos más satisfactorios lo cual redundará en un desempeño profesional de calidad y en la construcción de saberes de excelencia. De acuerdo con Rinaudo (2006) no hay combinación más perfecta que un estudiante motivado para aprender y un profesor amante de su materia, con buenas herramientas para enseñarla.

En este contexto, los factores motivacionales juegan un papel relevante en el control, organización y dirección de la conducta del estudiante ante el aprendizaje, pues ellos contribuyen a desarrollar sus capacidades, superar sus limitaciones y atender a sus intereses. Barriga y Hernández (2002) destacan que estos se relacionan e influyen en la manera de pensar del estudiante, las metas que establece, el esfuerzo y persistencia que manifiesta, las estrategias de estudio que emplea y el tipo de consecuencias asociadas al aprendizaje resultante.

El presente estudio se ha estructurado siguiendo un orden metodológico en los que se esboza de manera sucinta el fundamento teórico, el desarrollo y los resultados que se obtendrán, como producto de un arduo análisis estadístico e interpretativo.

\section{Literatura}

\subsection{Factores motivacionales}

Los factores motivacionales involucran los sentimientos relacionados con el crecimiento personal, el reconocimiento profesional y las necesidades de autorrealización que dependen de las tareas que el individuo realiza y el aprendizaje es la fuente principal para lograr el desarrollo de la persona y la sociedad. Es importante señalar, que la mayoría de los estudiantes del Departamento de Ciencias Económicas y Administrativas 
de la Facultad Regional Multidisciplinaria de Chontales de la UNAN- MANAGUA (UNAN - FAREM - Chontales), muestran una desmotivación hacia las actividades de aprendizaje, las cuales tienden a convertirse en un compromiso y obligación, más que en un disfrute por sí mismo, la motivación no se dirige hacia el aprendizaje.

En este contexto, y sustentado en los resultados encontrados en varias investigaciones hechas en esta área temática (Garbanzo 2007, Montero, Villalobos, \& Valverde, 2007), los factores que influyen en la motivación pueden clasificarse siguiendo diversos criterios que dependen fundamentalmente de la propia persona (factores personales), del contexto que envuelve al sujeto e influye en él (factores del contexto académico y factores sociales e institucionales) (Barriga \& Hernández, 2010) y que a continuación, veremos de manera sucinta.

\subsubsection{Factores Personales}

Las metas son normas específicas de desempeño que aumentan la motivación y la autoeficacia porque es fácil evaluar el progreso hacia la meta (Schunk, 2012). Éstas pueden ser de muy distintos tipos y su importancia como factor determinante del interés y el esfuerzo que el estudiante pone en su aprendizaje varía en función de la edad, sexo, experiencias académicas y el contexto sociocultural. Así, puede afirmarse que dentro de los motivos principales que animan a los estudiantes a estudiar están aprender, alcanzar el éxito; evitar el fracaso; ser valorado por sus profesores, padres o compañeros, u obtener muy diversos tipos de recompensas. Las atribuciones son lo que se percibe como causa de los resultados. Por ejemplo, si un estudiante atribuye a la suerte el aprobado en un examen, o bien considera que ha aprobado gracias al esfuerzo que ha realizado, es evidente que esta atribución influirá en la manera de afrontar un nuevo examen.

Según la causa que el estudiante atribuya, así va a incidir sobre el autoconcepto $2 /$, su confianza en sus capacidades y en las conductas futuras de logro académico, es así como la mayoría de las variables personales que orientan la motivación parten de las creencias y percepciones que el individuo tiene sobre aspectos cognitivos. Las expectativas son uno de los determinantes motivacionales más importantes que influyen en la consecución efectiva del éxito o fracaso que tiene la persona en un aspecto de su vida. En definitiva, las actitudes, percepciones, expectativas y representaciones que tenga el estudiante de sí mismo, de la tarea a realizar y de las metas que pretende alcanzar constituyen factores que guían y dirigen la conducta del estudiante. Pero también hay que tener en cuenta las variables externas procedentes del contexto en el que éstos se desenvuelven.

2 / El autoconcepto se define como el conjunto de percepciones y creencias que una persona posee sobre sí misma. 


\subsubsection{Factores Pedagógicos}

La manera en que se organice la enseñanza en el aula y la actitud del docente serán determinantes para el desarrollo de la motivación de los estudiantes y para conseguir la mayor eficacia en los objetivos de aprendizaje (Montero et al. 2007). En este contexto, Alonso (1997) plantea lo siguiente:

Él - docente - es quien decide qué información presentar, cuándo y cómo hacerlo; qué objetivos proponer; qué actividades planificar; qué mensajes dar a los alumnos, antes, durante y después de las diferentes tareas; cómo organizar las actividades -de forma individual, cooperativa o competitiva-; qué y cómo evaluar; cómo comunicar a los alumnos los resultados de las evaluaciones; qué uso hacer de la información recogida (p.12). Por ello el docente ejerce una influencia decisiva, ya sea consciente o inconscientemente, en lo que los estudiantes quieran saber y sepan pensar. Asimismo, Page (1990, citado por Montero et al., 2007) destaca que "su capacidad para comunicarse, las relaciones que establece con el alumno(a) y las actitudes que adopta hacia él, juegan un papel determinante tanto en el comportamiento como en el aprendizaje del (la) estudiante" (p. 218).

Se debe agregar que, en este factor se incluyen las diferentes estrategias de enseñanza utilizadas por el docente, los métodos de evaluación y materiales didácticos, así como el tiempo dedicado por los profesores a la preparación de sus clases.

\subsubsection{Factores sociodemográficos}

Además de los factores personales y pedagógicos, existen otros factores que también influyen en su actitud frente al estudio y, por tanto, en su motivación para aprender. Se trata de la influencia de la familia y de la sociedad, en general, en el desarrollo y mantenimiento de la motivación de los estudiantes para aprender, tal y como se examinan a continuación:

El entorno familiar se entiende como un conjunto de interacciones propias de la convivencia familiar, que afectan el desarrollo del individuo, manifestándose también en la vida académica (Garbanzo, 2007), entre los factores familiares podríamos citar el nivel socioeconómico, educativo y cultural de los padres o adultos responsables del estudiante, la valoración que la familia tiene de la cultura, la educación y el conocimiento, la atención y ayuda prestada a sus hijos, las expectativas de los padres respecto al futuro de sus hijos, el control ejercido sobre ellos, el seguimiento de sus actividades académicas, el conocimiento de las amistades del hijo, las actividades de ocio promovidas por la familia, las relaciones personales afectivas entre los miembros de la familia, y otros. 
Los factores sociales son aquellos que interactúan con la vida académica del estudiante y pueden influir en su desempeño académico, podemos señalar como los más importantes el entorno social en que se desenvuelve el sujeto, la valoración social de la cultura, los aprendizajes, la educación o los títulos académicos, los valores dominantes en la sociedad, los modelos dominantes de paternidad y de relaciones entre padres e hijos, los medios de distracción y ocio de que disponen los jóvenes, y otros.

Las Variables demográficas se refieren a las condiciones como la zona geográfica de procedencia, zona geográfica en la que vive el estudiante en época lectiva entre otros. Por ejemplo: la variable sexo del (la) estudiante fue considerada en la población bajo estudio, ya que proporciona información relevante para diversos análisis demográficos, sociales y económicos.

\subsubsection{Factores Institucionales}

Esta categoría es definida por Latiesa (en Montero et al.,2007) como características estructurales y funcionales que difieren en cada institución, y su grado de influencia confiere a la universidad peculiaridades propias, dentro de estos se encuentran: los horarios de las materias, los tamaños de los grupos, dificultad de algunas materias, aspectos relacionados con la carrera, el ambiente institucional y las relaciones estudiante-profesor, que influyen en el aprendizaje de los estudiantes.

En síntesis, es importante señalar que, dada la complejidad del tema, es posible que valiosos indicadores no hayan sido abordados y, que en las relaciones que se establecen entre los distintos factores, no es posible hablar de una generalización, pues los resultados podrían variar según el conjunto de interacciones causales, las poblaciones que se estudien y sus contextos.

\section{Metodología de la investigación}

El presente estudio fue realizado en el período del II semestre del año 2017 y I semestre del 2018 que consiste en una investigación mixta y de tipo descriptiva, ya que se reseñan las características de la relación que existe entre los factores que determinan la motivación por aprender.

La población está compuesta por 266 estudiantes. La muestra es de 78 estudiantes de los cuartos y quintos años de las carreras de Administración de Empresas, Contaduría Pública y Finanzas y Mercadotecnia del Departamento de Ciencias Económicas y Administrativas de la Facultad Regional Multidisciplinaria, Chontales (FAREM Chontales) en las modalidades: diurno, por encuentro y profesionalización. Se utilizó la encuesta como instrumento. La validez se obtuvo a través de la técnica del juicio de expertos, y la confiabilidad se determinó aplicando la prueba de dos mitades. 


\section{Resultados}

Más de la mitad de los estudiantes (53,8\%) manifestaron que sus padres siempre contribuyen al buen desempeño de sus estudios y con expectativas realistas. Solo una minoría respondió lo contrario.

\section{Figura 1. Contribución de los padres en el buen desempeño del estudiante}

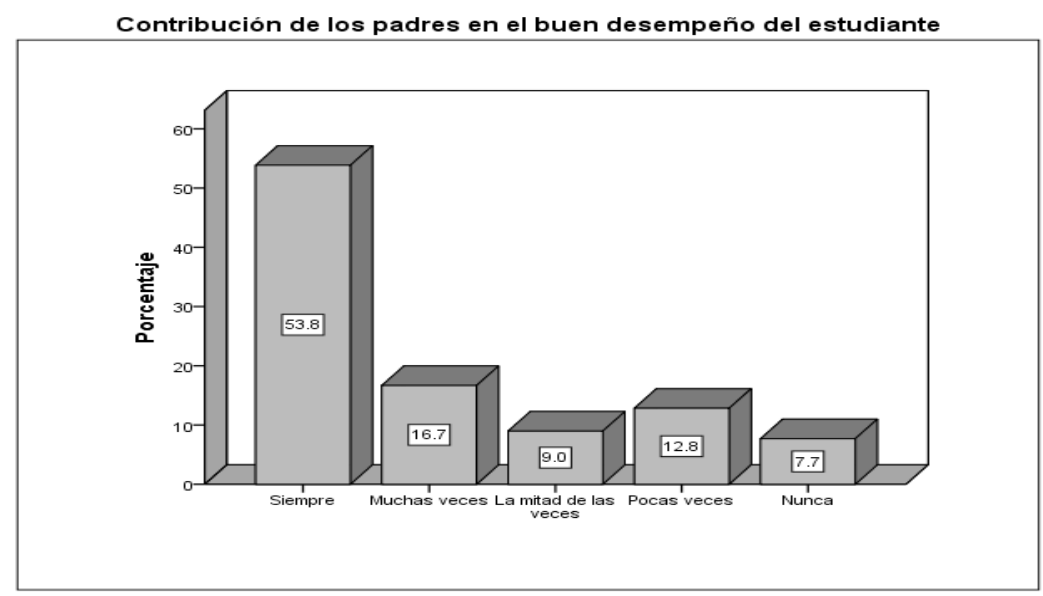

En la tabla 1. se observa que más de la mitad de los estudiantes $(59,0 \%)$ han reprobado más de una asignatura que dentro de los motivos que más influyeron, en orden de prioridad, destacan los siguientes: el 50,0\%, "no entendía las explicaciones del profesor", el 30,4\%, "la mala relación con el grupo de clase" y el 17,4\% afirmó que "la metodología del profesor era inadecuada".

Tabla 1. Motivos que influyeron para que el estudiante reprobará en alguna asignatura

\begin{tabular}{|l|c|}
\hline \multicolumn{1}{|c|}{ Motivos } & $\begin{array}{c}\text { Reprobación de alguna asignatura } \\
\text { Sí }\end{array}$ \\
\hline No entendía las explicaciones del profesor & $50.0 \%$ \\
\hline No tenía interés por la asignatura & $17.4 \%$ \\
\hline La metodología del profesor era inadecuada & $30.4 \%$ \\
\hline $\begin{array}{l}\text { Las malas relaciones con el grupo de clase influyó } \\
\text { para que dejara la asignatura }\end{array}$ & $6.5 \%$ \\
\hline Tenía malas relaciones con el profesor & $2.2 \%$ \\
\hline Falta de tiempo para estudiar & $10.9 \%$ \\
\hline Inasistencia a clases & $10.9 \%$ \\
\hline Problemas de Salud & $2.2 \%$ \\
\hline
\end{tabular}


Es válido aclarar que se hizo uso de la frecuencia de conjuntos de respuestas múltiples, donde solo se tomó a aquellos estudiantes que han reprobado alguna asignatura y que suman 46 de una muestra total de 78 estudiantes.

\begin{tabular}{|c|c|}
\hline Motivos & Total \\
\hline Prestigo de la universidad & $75.6 \%$ \\
\hline Tradicción familiar & $10.3 \%$ \\
\hline Requisito de matricula & $25.7 \%$ \\
\hline Oportunidad de mejorar mi estatus social & $50.0 \%$ \\
\hline Reconocimiento social de la carrera & $46.1 \%$ \\
\hline Posibilidad de trabajo & $62.8 \%$ \\
\hline Modalidad de estudios (Diurnos, Nocturno, Sabatino & $64.2 \%$ \\
\hline Gusto por los estudios & $47.5 \%$ \\
\hline Ingresos económicos & $34.7 \%$ \\
\hline
\end{tabular}

En la tabla 2, se observa que dentro de los motivos que influyeron en la elección de la carrera que estudian destacan, en orden de prioridad; el "prestigio de la Universidad", "la modalidad de estudios (diurno, nocturno y sabatino)" y las "posibilidades de trabajo".

\begin{tabular}{|c|l|c|}
\hline \multirow{3}{*}{ Grado de satisfacción } & \multicolumn{2}{|c|}{ Tabla 3. Grado de Satisfacción } \\
\cline { 2 - 3 } & \multicolumn{1}{|c|}{ Escala } & Total \\
\cline { 2 - 3 } & Altamente satisfecho & $35.6 \%$ \\
\cline { 2 - 3 } & Medianamente satisfecho & $48.9 \%$ \\
\cline { 2 - 3 } & Muy poco satisfecho & $14.1 \%$ \\
\cline { 2 - 3 } & Nada satisfecho & $1.4 \%$ \\
\hline \multicolumn{2}{|c|}{ Total } & $100.00 \%$ \\
\hline
\end{tabular}

A juzgar por los resultados de la tabla 3, con respecto al grado de satisfacción que tiene el estudiante sobre los siguientes aspectos: "metodologías de enseñanza", "técnicas de evaluación", "las relaciones profesor- estudiante", "mis avances académicos", "mi método de estudio", "los servicios de la universidad", "el ambiente universitario" y "mi nivel de integración", casi la mitad de las respuestas (48,9\%) fueron valoradas por el estudiante como "medianamente satisfecho", seguido de "altamente satisfecho" con el 35,6\%, sólo un 15,5\% dijo sentirse "muy poco satisfecho" o "nada satisfecho".

\section{Discusión y conclusiones}

Entre los factores que determinan la motivación por aprender destacan los personales y pedagógicos. Cabe destacar que los padres son un pilar fundamental en la formación académica de sus hijos y es necesario darles seguimiento continuo y moderado en compañía de la universidad. Esto realmente es un aspecto importante 
en el asunto de la motivación ya que hay un buen porcentaje de estudiantes que no cumplen con sus expectativas y que tampoco están satisfechos; se enfatizan más en los aprendizajes mecánicos, con una clara incidencia negativa sobre la comprensión, por tanto, el rendimiento académico que obtienen no es el esperado. En este contexto, el profesor debe ofrecer la dirección y la guía pertinentes hacia las tareas académicas y desarrollar diversas estrategias didácticas que estimulen el aprendizaje autónomo.

\section{Lista de referencias}

Alonso Tapia, J. (1997). Motivar para el aprendizaje. Teorías y estrategias. Barcelona, España: Edebé.

Arancibia C., V., Herrera P., P., \& Strasser S, K. (2008). Manual de Psicología Educacional. Santiago, Chile: Ediciones Unversidad Católica de Chile.

Barriga Arceo, F. D., \& Hernández Rojas, G. (2002). Estrategias docentes para un aprendizaje significativo. Una interpretación constructivista (Segunda ed.). México, D.F., México: MCGraw-Hill Interamericana.

Bernal, C. (2010). Metodología de la investigación. (Tercera ed.). Colombia: PEARSON EDUCACIÓN.

Garbanzo Vargas, G. M. (12 de Agosto de 2007). Factores asociados al rendimiento académico en estudiantes universitarios, una reflexión desde la calidad de la educación superior pública. Revista Educación, 1(31), 43-63. Recuperado el 19 de Noviembre de 2016.

Hernández Sampieri, R., Fernández Collado, C., \& Baptista Lucio, M. d. (2014).

Martínez-Salanova, E. (s.f.). Obtenido de uhu.es: http://www.uhu.es/cine.educacion/ didactica/oo83motivacion.htm

Montero Rojas, E., Villalobos Palma, J., \& Valverde Bermúdez, A. (2007). Factores institucionales, pedagógicos, psicosociales y sociodemográficos asociados al rendimiento académico en la unviersidad de Costa Rica: Un análisis multinivel. RELIEVE, 13(2), 215-234.

Metodología de la Investigación (Sexta ed.). México D.F.: McGraw-Hill Education.

Ormrod, J. E. (2005). Aprendizaje Humano (Cuarta ed.). Madrid, España: PEARSON EDUCACIÓN, S.A. 
Rinaudo, M. C., De la Barrera, M. L., \& Donolo, D. S. (1997). Motivación para el aprendizaje en alumnos universitarios. Revista Electrónica de Motivación y Emoción (REME), IX(22), 1-19.

Schunk, D. H. (2012). Teorías del Aprendizaje. Una perspectiva educativa (Sexta ed.). México: Pearson Educación.

Woolfolk, A. (2010). Psicología Educativa (Decimoprimera ed.). México, México: PEARSON EDUCACIÓN. 